\section{International Scientific Journal Theoretical \& Applied Science}

p-ISSN: 2308-4944 (print) e-ISSN: 2409-0085 (online)

Year: $2017 \quad$ Issue: $05 \quad$ Volume: 49

Published: 3.05.2017 http://T-Science.org

SECTION 7. Mechanics and machine construction.
Nikolay Yuryevich Loginov

Associate Professor of the Department «Equipment and technology of machine-building production»" FSBEI HPE «Togliatti State University», Russia

Vadim Anatolyevich Gulyaev Associate Professor of the Department «Equipment and technology of machine-building production»» FSBEI HPE «Togliatti State University», Russia Colmy@tltsu.ru

\title{
DETECTION SYSTEM OF DEFECTS AUTOMOBILE REAR VIEW MIRRORS
}

\author{
Abstract: In this report we describe the system of the automated contactless control of optical defects of \\ automobile rear-view mirrors. \\ Key words: contactless control, automobile mirrors, control of defects. \\ Language: Russian \\ Citation: Loginov NY, Gulyaev VA (2017) DETECTION SYSTEM OF DEFECTS AUTOMOBILE REAR \\ VIEW MIRRORS. ISJ Theoretical \& Applied Science, 05 (49): 28-31. \\ Soi: http://s-o-i.org/1.1/TAS-05-49-6 Doi: crossef https://dx.doi.org/10.15863/TAS.2017.05.49.6
}

\section{СИСТЕМА ОПРЕДЕЛЕНИЯ ДЕФЕКТОВ АВТОМОБИЛЬНЫХ ЗЕРКАЛ ЗАДНЕГО ВИДА}

Аннотация: В данной статье рассматривается система автоматизированного бесконтактного контроля оптических дефектов автомобильных зеркал заднего вида.

Ключевые слова: бесконтактный контроль, автомобильные зеркала, контроль дефектов.

\section{Introduction}

В современном автомобилестроении предъявляются жесткие требования к качеству выпускаемой продукции, что обусловлено не только обеспечением безопасности эксплуатации автомобилей, но и огромной конкуренцией на рынке. Выполнение этих требований возможно только путем увеличения требований к контролю и к контрольным системам всех элементов автомобиля. Рост объемов производства приводит к необходимости автоматизации всех процессов на производстве, что ставит проблему организации контроля в ряд наиболее актуальных для автомобилестроения.

Автоматизация контроля зеркал заднего вида подразумевает, прежде всего, поиск и определение дефектов, которые ухудшают обзорность с места водителя. Перечень этих дефектов достаточно большой [1]. Это могут быть включения, пороки формирования, механические повреждениями. Ocобое внимание уделяется наиболее распространенным дефектам сколам и потертостям. Данные дефекты могут быть изначально незначительными по размерам и малозаметными, но при определенных условиях могут разрастаться. Однако, в соответствии с различными стандартами наличие таких дефектов допускается в зависимости от места их расположения и размеров.

\section{Materials and Methods}

Контроль дефектов зеркал на действующих предприятиях на данный момент чаще всего не автоматизирован и выполняется специально обученным контролером с применением простейших оптических приспособлений или без них. В таком случае дается только качественная оценка, т.е. есть дефект или он отсутствует. Кроме того, такой метод контроля обладает низкой достоверностью в виду субъективности восприятия оптической информации каждым отдельным человеком [2]. Еще одним существенным недостаткам метода является его незначительная производительность. В случае применения простейших оптических приспособлений можно получать в ряде случаев количественную оценку дефектов. Недостаток приборов данного класса заключается в том, что они требуют большой концентрации внимания и сильно нагружают зрение операторов. Поэтому 


\begin{tabular}{|c|c|c|c|c|c|c|}
\hline Impact Factor: & $\begin{array}{l}\text { ISRA (India) } \\
\text { ISI (Dubai, UAE } \\
\text { GIF (Australia) } \\
\text { JIF }\end{array}$ & $\begin{array}{r}=1.344 \\
=0.829 \\
=0.564 \\
=1.500\end{array}$ & $\begin{array}{l}\text { SIS (USA) } \\
\text { PИНЦ (Russia } \\
\text { ESJI (KZ) } \\
\text { SJIF (Moroccc }\end{array}$ & $\begin{aligned} &= 0.912 \\
&= 0.234 \\
&= \mathbf{3 . 8 6 0} \\
&=\mathbf{2 . 0 3 1}\end{aligned}$ & $\begin{array}{l}\text { ICV (Poland) } \\
\text { PIF (India) } \\
\text { IBI (India) }\end{array}$ & $\begin{array}{l}=6.630 \\
=1.940 \\
=4.260\end{array}$ \\
\hline
\end{tabular}

такое решение не является оптимальным, т.к. представляет опасность для здоровья оператора.

Автоматизированный контроль решает все перечисленные недостатки [3]. Как правило, системы данного класса представляют собой программно-аппаратные комплексы, построенные на базе систем технического зрения. На данный момент такого рода системы контроля дефектов зеркал серийно промышленностью не выпускаются. В связи с этим на большинстве предприятий используется неавтоматизированный контроль.

Построение такой системы было решено производить на основе оптико-цифрового модуля, персонального компьютера и специального программного обеспечения, разработанного на базе теории цифровой обработки изображений $[4,5,6]$.

Схема спроектированного стенда представлена на рисунке 1. Представленная установка состоит из следующих основных элементов:

1.Цифровая ПЗС фотокамера.

2.Оправа ПЗС фотокамеры.

3.Корпус, оснащенный источником монохроматического света.

4.ПЭВМ и программное обеспечение.

5.Монотонное полотно.

6.Стол.

7.Контролируемый образец.

8.Оправа для зеркала.

В процессе работы стенда изображение зеркала заднего вида с дефектами регистрируется цифровой фотокамерой. Далее изображение, представляющее собой цифровой фотоснимок попадает на обработку в ПЭВМ, где производится его обработка в специальном программном обеспечении. После чего выдается информация о наличии дефектов в контролируемом изделии, месте их расположения и размере.

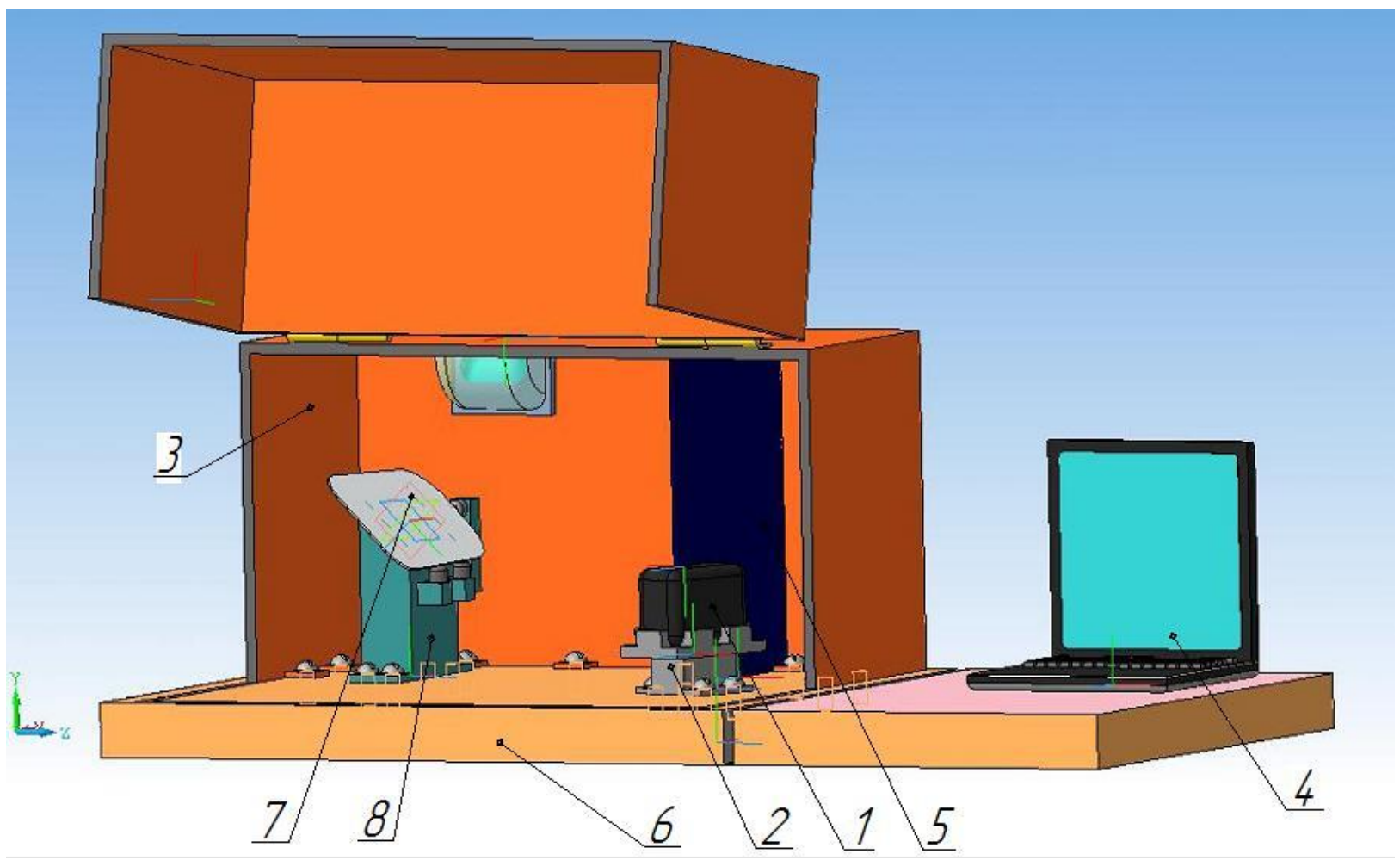

Рисунок 1 - Схема стенда контроля оптических дефектов.

Рассмотрим более подробно работу стенда.

Контролируемое зеркало заднего вида помещается в оправку 8. ПЗС фотокамера 1 заранее устанавливается в оправке 2 на столе. При этом камера должна быть настроена заранее так, чтобы обеспечивалось перекрытие зеркала монотонным полотном 5.

Полученное изображение подвергается обработке на ПЭВМ 4. Сначала с изображения удаляются «шумы». С этой целью могут быть использованы известные алгоритмы [7].

Затем необходимо подвергнуть изображение бинаризации, тем самым, выделив на изображении дефекты.

Существуют различные методы бинаризации изображений. В данном случае достаточно применения одного из самых простых - пороговой обработки. [8] Данный метод основан на различии средней яркости 
однородных участков изображения. Проведение соответствующего преобразования каждой точки изображения должно выполняться в соответствии с правилом:

$$
y(i, j)=\left\{\begin{array}{l}
y_{0}, \text { при } x(i, j) \leq x_{0} \\
y_{1}, \text { при } x(i, j)>x_{0}
\end{array}\right.
$$

где $x_{0}$ - единственный параметр обработки, называемый порогом. Уровни выходной яркости $y_{0}$ и $y_{1}$ могут быть произвольными.

Далее производим обнаружение дефектов путем вычитания полученного изображения из эталона, т.е. получения бинарной разности [9].

$$
\begin{aligned}
& e_{i, j}=\left\{\begin{array}{l}
Z\left(p_{i, j}-s(A, \mu, \lambda)_{i+Y, j+X}\right) \\
0
\end{array},\right. \\
& l_{i, j}=\left\{\begin{array}{l}
Z\left(s(A, \mu, \lambda)_{i+Y, j+X}-p_{i, j}\right) \\
0
\end{array}\right.
\end{aligned}
$$

где

$$
Z(x)=\left\{\begin{array}{ll}
x, & x>0 \\
0 & \text { в } \text { противном случае }
\end{array},\right.
$$

Наличие «лишних» или «недостающих» фрагментов говорит о наличии на данном участке дефекта. Далее оценивается область на допустимость наличия дефекта [9].

В ряде случаев необходимо определить не только наличие дефектов, но и их геометрические характеристики. Решению данной задачи посвящено большое количество литературы. В частности можно применять алгоритмы рассмотренные в [10].

\section{Conclusion}

Одним из преимуществ данной системы является возможность ее подключения к компьютерной сети предприятия. Это позволит своевременно получать информацию о дефектах, проводить ее статистическую обработку и вносить соответствующие коррективы в технологический процесс изготовления зеркал заднего вида автомобиля. Другое немало важное преимущество спроектированной системы заключается в том, что из процесса контроля полностью исключается человеческий фактор, что резко повышает достоверность получаемой информации. Кроме того, данная система дает не только качественную, но и количественную информацию о дефектах, что при применении традиционных методах контроля затруднительно.

\section{References:}

1. Zhilin AA, Soldatov AA, Guliaev VA (2004) Dinamicheskoe nagruzhenie kak metod otsenki dopustimykh vnutrennikh napriazhenii termoobrabotannykh opticheski aktivnykh elementov // Izvestiia Volgogradskogo gosudarstvennogo tekhnicheskogo universiteta 2004. №1. p. 35-36.

2. Kozlov AA (2005) Povyshenie kachestva kontrolia zazorov kuzova avtomobilia putem avtomatizatsii protsessa / A. A. Kozlov avtoreferat dissertatsii na soiskanie uchenoi stepeni kandidata tekhnicheskikh nauk / Moskovskii gosudarstvennyi tekhnologicheskii universitet. Toliatti.

3. (2009) Osnovy rascheta i proektirovaniia sistem avtomaticheskogo upravleniia v mashinostroenii: Ucheb. posobie / O. I. Drachev, D. A. Rastorguev, A. A. Soldatov, A. G. Skhirtladze - Staryi Oskol.

4. Gruzman IS (2002) TSifrovaia obrabotka izobrazhenii $\mathrm{v}$ informatsionnykh sistemakh Ucheb. posobie / I. S. Gruzman, V. S. Kirichuk,
V. P. Kosykh - Novosibirsk: Izd-vo NGTU, $352 \mathrm{p}$.

5. Otsu N (1979) A Threshold Selection Method from Gray-Level Histograms, IEEE Transactions on Systems, Man, and Cybernetics, Vol. 9, No. 1, 1979, p. 62-69.

6. Gordeev AV, Loginov NI (2015) Optimizatsiia tekhnicheskikh parametrov pri reshenii inzhenernykh zadach // Vektor nauki Toliattinskogo gosudarstvennogo universiteta. 2015. №4(34). p. 25-30.

7. Kozlov A, Valentiev V (2013) Detection of defects by the Otsu method at control of automobile rear-vew mirrors / Kozlov A., Valentiev V. -V sbornike: PRESSING ISSUES AND PRIORITIES IN DEVELOPMENT OF THE SCIENTIFIC AND TECHNOLOGICAL COMPLEX B\&M Publishing Research and Publishing Center «Colloquium»; Science editor: A. Burkov. San Francisco, California, USA, 2013. p. 109-112. 
\begin{tabular}{l|lrl|l|ll} 
& ISRA (India) & $=\mathbf{1 . 3 4 4}$ & SIS (USA) & $=\mathbf{0 . 9 1 2}$ & ICV (Poland) & $=\mathbf{6 . 6 3 0}$ \\
Impact Factor: & ISI (Dubai, UAE) $=\mathbf{0 . 8 2 9}$ & PUHL (Russia) $=\mathbf{0 . 2 3 4}$ & PIF (India) & $=\mathbf{1 . 9 4 0}$ \\
& GIF (Australia) & $\mathbf{0 . 5 6 4}$ & ESJI (KZ) & $=3.860$ & IBI (India) & $=\mathbf{4 . 2 6 0}$ \\
& JIF & $=\mathbf{1 . 5 0 0}$ & SJIF (Morocco) & $=\mathbf{2 . 0 3 1}$ & & \\
\hline
\end{tabular}

8. Soldatov AA, Guliaev VA, Zhilin AA, Belous DV (2004) Razrabotka avtomatizirovannoi sistemy beskontaktnogo kontrolia geometricheskikh parametrov kuzova avtomobilia // Izvestiia Volgogradskogo gosudarstvennogo tekhnicheskogo universiteta. 2004. №1. p. 46-48.

9. Kozlov AA (2005) Povyshenie kachestva kontrolia zazorov kuzova avtomobilia putem avtomatizatsii protsessa / A. A. Kozlov - dissertatsiia na soiskanie uchenoi stepeni kandidata tekhnicheskikh nauk / Toliatti, 2005

10. Kozlov AA (2015) Ekspress-analiz mikrotopografii poverkhnosti pri plakirovanii gibkim instrumentom // V sbornike: Teplofizicheskie i tekhnologicheskie aspekty povysheniia effektivnosti mashinostroitelnogo proizvodstva. Trudy IV mezhdunarodnoi nauchno-tekhnicheskoi konferentsii (Reznikovskie chteniia). p. 261-264. 